from the endoscopy documentation, and a patient comfort score was completed by the patient in the recovery area. The endoscopists and nurses were unaware that the comfort data was going to be studied. The wilcoxon matched pairs signal rank statistical test was used to look for comfort score differences between the groups.

Results A total of 139 procedures were included in the analysis. The OGD mean comfort scores were: endoscopist 1.3 (SEM, 0.1), nurse 1.6 (SEM, 0.1), patient 1.4 (SEM, 0.1). A significant difference was found between the endoscopist and nurse comfort scores $(\mathrm{p}<0.01)$. No significant difference was found for FS comfort scores: endoscopist 1.8 (SEM, 0.1), nurse 1.6 (SEM, 0.1 ), and patient 1.8 (SEM, 0.2). For colonoscopy, the mean scores were: endoscopist 1.7 (SEM, 0.1), nurse 2.1 (SEM, 0.2), patient 1.6 (SEM, 0.2). A significant difference was found between patient and nurse comfort scores $(\mathrm{p}<0.01)$, but not between the patient and endoscopist comfort scores.

Conclusion Endoscopy nurses gave a higher comfort score (more discomfort) than patients and endoscopists for OGD and colonoscopy, with no difference between the groups for FS comfort scores. These results suggest that the perception of procedure related discomfort differs between these three groups, particularly between endoscopists and nurses. As patient experience is a key aspect of endoscopy service quality, it is important to recognise that there are differences between the perceived comforts levels between the endoscopist, the nurse and the patient.

Disclosure of Interest None Declared.

\section{PWE-041 MANAGEMENT OF LARGE COLONIC POLYPS IN A BOWEL CANCER SCREENING PROGRAMME}

HY Lee*, W Gashau, R Willert. Department of Endoscopy, Manchester Royal Infirmary, Manchester, UK

\subsection{6/gutjnl-2014-307263.301}

Introduction Bowel cancer is the third most common cancer in the United Kingdom forming up to $13.6 \%$ of all newl1y diagnosed cancers (1). Bowel cancer screening colonoscopy allows early polyp detection at a curable stage. Complete resection and follow-up of large polyps is crucial to prevent malignant progression.

The aim of this study was to review the management of polyps with diameters $\geq 2 \mathrm{~cm}$, particularly of sessile polyps, to assess the enbloc resection rates, completeness of resection using endoscopic mucosal resection (EMR) vs surgery and the incidence of malignant polyps.

Methods Patients were identified retrospectively from a regional bowel screening programme database. Details of index colonoscopy including polyp characteristics, method of resection and complications were recorded. Histology results were reviewed for all polyps. Outcomes from follow-up endoscopic surveillance were analysed.

Results One hundred and fifty-eight patients (102 males, 56 females, mean age 66.2 years) with polyps $\geq 2 \mathrm{~cm}$ were identified from 2182 screening colonoscopies from January 2010 to August 2013. Caecal intubation rate was $96.8 \%$ in this group.

Largest polyp size for each patient ranged from 20 to $60 \mathrm{~mm}$ (mean $26.6 \mathrm{~mm}$ ). The incidence of adenocarcinoma was $11.9 \%$ $(\mathrm{n}=19)$, all located within the left colon, with 12 requiring surgical resection.

One hundred thirty nine patients $(\mathrm{n}=139)$ had 155 nonmalignant large polyps, mostly tubulovillous or villous histology $(\mathrm{n}=110,79 \%)$.
Thirty-six patients had 37 sessile polyps which underwent primary resection by EMR $(n=26)$ or surgery $(n=11)$.

Polyp diameter was larger in the surgery group with mean polyp diameter of 40.4 vs. $28.0 \mathrm{~mm}(\mathrm{p}<0.05)$.

EMR enbloc resection rate was $11.5 \%(\mathrm{n}=3$ out of 26$)$. Completeness of excision was $38.4 \%(\mathrm{n}=10)$ at 3 months and $92.3 \%(\mathrm{n}=24)$ at 1 year. EMR complications included 1 perforation, 1 post polypectomy syndrome and 1 bleed.

Surgical resection included: anterior resection in 2, TEMS excision in 7 and right hemicolectomy in 3 .

Conclusion Sessile polyps $\geq 2 \mathrm{~cm}$ are relatively uncommon in an asymptomatic bowel cancer screening programme (37 in 2182 colonoscopies). They can be successfully resected by EMR without recurrence in $92.3 \%$ at 1 year providing a 3 month site check is performed in all piecemeal polypectomies.

\section{REFERENCE}

1 Cancer for National Statistics 2010. Office for National Statistics. http://www.ons. gov.uk/ons/dcp171778_263537.pdf

Disclosure of Interest None Declared.

\section{PWE-042 ASSOCIATION BETWEEN MIDAZOLAM DOSE AND CAECAL INTUBATION RATE AT COLONOSCOPY}

J Boyd*, L Lee, S Lanzon-Miller. Milton Keynes NHS Foundation Trust, Milton Keynes, UK

\subsection{6/gutjnl-2014-307263.302}

Introduction Midazolam is a short acting benzodiazepine that is commonly used for sedation during colonoscopy. There is no standard dose of midazolam; however, British Society of Gastroenterology guidelines suggest a maximum of $5 \mathrm{mg}$ with lower doses for elderly patients. Caecal intubation rate (CIR) is a commonly used performance indicator for colonoscopy. Data exploring the relationship between midazolam dose and CIR is limited. Methods A retrospective cohort study of all patients who had undergone a colonoscopy at Milton Keynes General hospital between January 2010 and December 2012. Patients were identified from the Endoscopy Unit database and their records were reviewed. Patient details, midazolam dose and depth of insertion were extracted into a standardised form. Caecal intubation was defined as insertion of the colonoscope to a point proximal to the ileocaecal valve so that the entire caecum could be visualised.

Results 6200 patients were included for analysis. The median age was 62 years and $49.4 \%$ were male. The mean midazolam dose was $1.9 \mathrm{mg}$. 1004 patients had a low dose of midazolam $(<2 \mathrm{mg}), 4618$ a standard dose $(2 \mathrm{mg})$ and 578 a high dose (>2 mg). The CIR in the low dose cohort was $83.6 \%$, in the standard dose cohort was $91.3 \%$ and in the high dose cohort was $78.7 \%$. Procedural discomfort was significantly greater in the high dose cohort. When patients with poor bowel preparation were removed from the cohort $(\mathrm{n}=5534)$, CIR was $85.2 \%$ in the low dose cohort vs. $92.1 \%$ in the standard dose cohort. Patients who received doses of midazolam $<2 \mathrm{mg}$ or $>2 \mathrm{mg}$

\begin{tabular}{llll}
\multicolumn{4}{l}{ Abstract PWE-042 Table 1} \\
\hline Midazolam dose & $<2 \mathrm{mg}$ & $2 \mathrm{mg}$ & $>2 \mathrm{mg}$ \\
$\mathrm{N}$ & $1004(16.2)$ & $4618(74.5)$ & $578(9.3)$ \\
Caecum positively identified & 839 & 4216 & 455 \\
CIR & $83.6 \%$ & $91.3 \%$ & $78.7 \%$ \\
P-value & $<0.001$ & - & $<0.001$ \\
\hline
\end{tabular}

